\title{
Expression patterns of programmed death ligand 1 correlate with different microenvironments and patient prognosis in hepatocellular carcinoma
}

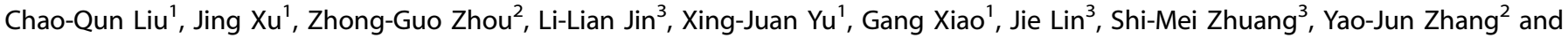 \\ Limin Zheng ${ }^{1,3}$
}

BACKGROUND: Recent clinical studies have suggested that programmed death ligand 1 (PD-L1) expression in a tumour could be a potential biomarker for PD-L1/PD-1 blockade therapies.

METHODS: To better characterise PD-L1 expression in hepatocellular carcinoma (HCC), we analysed its expression patterns in 453 HCC patients by double staining for CD68 and PD-L1 using the Tyramide Signal Amplification Systems combined with immunohistochemistry. We also investigated its correlation with clinical features, prognosis and immune status.

RESULTS: The results showed that PD-L1 expression on tumour cells (TCS) was negatively associated with patients' overall survival $(\mathrm{OS} ; P=0.001)$ and relapse-free survival (RFS; $P=0.006)$; however, $\mathrm{PD}-\mathrm{L} 1$ expression on macrophages (M $\varphi \mathrm{S})$ was positively correlated with OS $(P=0.017)$. Multivariate analysis revealed that PD-L1 expression on TCs and M $\varphi s$ were both independent prognostic factors for OS (hazard ratio $(H R)=1.168, P=0.004$ for TC-PD-L1; HR $=0.708, P=0.003$ for M $\varphi$-PD-L1). Further studies showed that M $\varphi$-PD-L1 $1^{+}$tumours exhibited an activated immune microenvironment, with high levels of $\mathrm{CD} 8^{+} \mathrm{T}$-cell infiltration and immune-related gene expression.

CONCLUSION: Our study provided a novel methodology to evaluate PD-L1 expression in the tumour microenvironment, which might help to select patients who would benefit from anti-PD-1/PD-L1 immunotherapies.

British Journal of Cancer (2018) 119:80-88; https://doi.org/10.1038/s41416-018-0144-4

\section{INTRODUCTION}

Hepatocellular carcinoma (HCC) is one of the most prevalent tumours, with a high disease incidence and high mortality worldwide. ${ }^{1}$ Roughly one third of patients are eligible for potential curative treatments, such as surgical resection, radiofrequency ablation, and liver transplantation. ${ }^{2}$ However, these therapeutic treatments often do not provide a complete cure, and half of the treated patients develop tumour recurrence within 3 years. ${ }^{3-5}$ Although adjuvant therapies, including transarterial chemoembolisation and adoptive transfer of cytokine-induced killer cells, can improve the overall survival (OS) in some patients with HCC, their effects on tumour regression remain limited.,

Immune checkpoint blockade therapy, which targets regulatory pathways in $T$ cells to unleash immunosuppression and restore antitumour immune responses, has been hailed as a major breakthrough in oncology and provides a new weapon against cancer. ${ }^{8,9}$ Agents targeting the programmed death ligand 1 (PDL1)/programmed cell death protein-1 (PD-1) immune checkpoint displayed impressive antitumour effects and durable responses on a fraction of patients with melanoma, bladder carcinoma, nonsmall-cell lung carcinoma, Hodgkin's lymphoma, and renal cell carcinoma. ${ }^{10-14}$ Durable responses have been reported recently in patients with advanced HCC treated with Nivolumab, a fully human immunoglobulin antibody that disrupts PD-1 immune checkpoint signalling. In this multicentre phase $1 / 2$ study, treatment of patients with advanced HCC with Nivolumab resulted in substantial tumour reductions and objective response rates of $15-20 \%$, irrespective of the line of therapy. ${ }^{15}$ Despite these encouraging results, a major challenge is to identify biomarkers that predict therapeutic responsiveness, which could be used to select patients who might benefit from the treatment.

Although PD-L1 is not yet established as a consistently reliable biomarker across tumour types, emerging evidence indicates that patients who have a higher proportion of tumour cells (TCS) or immune cells-expressing PD-L1 might achieve greater benefit. ${ }^{16-18}$ PD-L1 can be constitutively or inducibly expressed on both TCS and immune cells in the tumour microenvironment (TME). 8,19,20 Several clinical studies have observed that patients with PD-L1 ${ }^{+}$ TCs were more likely to benefit from checkpoint blockade therapies ${ }^{21,22}$; while some studies emphasised that immune cells-expressing PD-L1 correlated with patients' responses. ${ }^{16,23,24}$ Based on these findings, the US Food and Drug Administration has

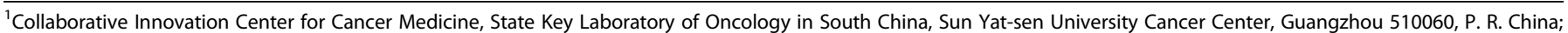

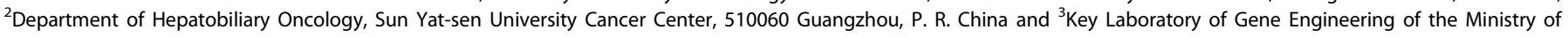
Education, State Key Laboratory of Biocontrol, School of Life Sciences, Sun Yat-sen University, Guangzhou 510275, P. R. China

Correspondence: Yao-Jun Zhang (zhangyuj@sysucc.org.cn) or Limin Zheng (zhenglm@mail.sysu.edu.cn)

These authors contributed equally: Chao-Qun Liu, Jing Xu.
}

Received: 5 February 2018 Revised: 9 May 2018 Accepted: 16 May 2018

Published online: 20 June 2018 
approved several PD-L1 assays to detect the overall PD-L1 expression level as a criterion to select patients for immunotherapy with anti-PD-1/PD-L1 antibodies. However, the levels of overall PD-L1 expression did not yield satisfactory results as a predictive biomarker to select patients who would benefit from treatment in clinical practice. ${ }^{25}$ Therefore, it is essential to optimise the detection method and understand the relevance of PD-L1 expression patterns in tumour tissues.

In the present study, we aimed to characterise PD-L1-expression in patients with $\mathrm{HCC}$ and investigate the correlation between the expression patterns of PD-L1 and patients' clinical features and prognosis. The TME features of different PD-L1 expression patterns were further explored using the Agilent human expression microarray to reveal their potential biological and clinical significance.

\section{MATERIALS AND METHODS}

Patients

All samples were anonymously coded in accordance with local ethical guidelines (as requested by the Declaration of Helsinki), with written informed consent and a protocol approved by the Review Board of Sun Yat-sen University Cancer Center.

Formalin-fixed, paraffin-embedded tissue from 453 patients with pathologically confirmed HCC, who had all received resection for tumours at the Sun Yat-sen University Cancer Center between 2006 and 2010, were enrolled in this study. The inclusion criteria used in patient enrolment were: Absence of anticancer therapies or distant metastasis before surgery; no concurrent autoimmune disease, HIV or syphilis; and availability of follow-up data. All patients underwent curative resection for HCC with the following intra-operative goals: A resection margin of at least $1 \mathrm{~cm}$, complete resection of all tumour nodules, and leaving the cut surface free of tumour (based on histological examination). Intraoperative ultrasound and post-surgical contrast-enhanced computed tomography were routinely used to ensure complete removal of HCC. $^{26,27}$ Macroscopic vascular invasion was determined using imaging or histology. Detailed characteristics of the patients enrolled in this study are summarised in Supplementary Table S1.

Patients were followed postoperatively at the outpatient clinic with regular surveillance for recurrence using the serum alphafetoprotein level, abdominal ultrasonography, and chest radiography, at 2-4-month intervals. ${ }^{26-28}$ When tumour recurrence or metastasis was suspected, further examinations, including computed tomography and hepatic angiography, were performed. Patients with confirmed recurrence received further treatment, including a second surgical resection, transarterial chemoembolisation, radiofrequency ablation, or percutaneous ethanol injection. The median follow-up was 52.7 months (range 1-132 months). Of the 453 patients who were examined during the follow-up period, 207 patients (45.7\%) died, 263 patients (58.1\%) were diagnosed with tumour recurrence, and 144 patients (31.8\%) remained alive without recurrence. Relapse-free survival (RFS) was defined as the interval between surgery and the first of recurrence or death, or between surgery and the last observation for patients without recurrence. OS was defined as the interval between surgery and death or between surgery and the last observation for surviving patients.

\section{Tissue microarray construction}

Tissues were used to construct a tissue microarray, as described previously. ${ }^{26}$ Briefly, tissue blocks containing the advancing edges of tumoural HCC tissue were used for tissue microarray (TMA) construction. Duplicate $1.0-\mathrm{mm}$ tissue cores were randomly taken from the tumoural area in the paraffin-embedded tissue blocks. TMAs containing the tissue cores were then cut into 4- $\mu \mathrm{m}$ sections for immunohistochemistry (IHC) staining.
Antibodies

Primary antibodies used in this study were as follows: a mouse antihuman CD68 monoclonal antibody (Dako, Carpinteria, CA, USA), a mouse anti-human CD33 monoclonal antibody (Leica Biosystems, Newcastle, UK), a rabbit anti-human CD11b monoclonal antibody (Abcam, Cambridge, MA, USA), a rabbit anti-human CD3 monoclonal antibody (Thermo Scientific, Rockford, IL, USA), a rabbit antihuman CD20 monoclonal antibody (ZSBio, Beijing, China), a mouse anti-human CD57 monoclonal antibody (ZSBio, Beijing, China), and a rabbit anti-human PD-L1 monoclonal antibody (clone: E1L3N $\mathrm{N}^{\mathrm{TM}}$; Cell Signaling Technology, Danvers, MA, USA).

\section{Immunohistochemistry}

Formalin-fixed, paraffin-embedded tissues were cut into $4-\mu \mathrm{m}$ thick sections and subjected to immunohistochemical analysis. Sections were dewaxed in xylene and rehydrated through a decreasing ethanol series. To diminish the activity of endogenous peroxidase, sections were placed in $0.3 \% \mathrm{H}_{2} \mathrm{O}_{2}$ for 10 min at room temperature. Heat-mediated retrieval was use for antigen retrieval. Slides were incubated overnight at $4{ }^{\circ} \mathrm{C}$ with different primary antibodies. Detection was performed using the EnVision Detection Systems (DakoCytomation, Carpinteria, CA, USA) following the manufacturer's instructions; sections were counter-stained with haematoxylin. Image acquisition was performed using an Eclipse advanced research microscope (Nikon, Melville, NY, USA).

Multiplex staining and multispectral imaging

To identify the cell subsets expressing PD-L1 in the TME, multiplex immunofluorescence staining was obtained using TSA Plus Fluorescence Kits (PerkinElmer, Foster City, CA, USA) combined with IHC (TSA-IHC). Different primary antibodies were sequentially applied, followed by horseradish peroxidase-conjugated secondary antibody incubation and tyramide signal amplification. The slides were microwave heat-treated after each TSA operation. Nuclei were stained with $4^{\prime}-6^{\prime}$-diamidino-2-phenylindole (DAPI, Thermo Scientific) after all the human antigens had been labelled.

To obtain multispectral images, the stained slides were scanned using the Vectra System (PerkinElmer), which captures the fluorescent spectra at 20-nm wavelength intervals from 420 to $720 \mathrm{~nm}$ with identical exposure time; the scans were combined to build a single stack image.

For colocalisation analysis, images were acquired using a laser confocal microscope (Olympus, Essex, UK) and analysed using FV10-ASW Viewer software (Olympus).

Image analysis

Images of unstained and single-stained sections were used to extract the spectrum of autofluorescence of tissues and each fluorescein, respectively. The extracted images were further used to establish a spectral library required for multispectral unmixing by Nuance and InForm image analysis software (PerkinElmer). Using this spectral library, we obtained reconstructed images of sections with the autofluorescence removed.

To define PD-L1 ${ }^{+}$tumours, specimens displaying unequivocal membranous or cytoplasmic PD-L1 staining were classified as positive. In the tumour nest, PD-L1-expressing $\mathrm{CD}^{+} 8^{+} \mathrm{M} \varphi s$ showed a membranous staining pattern and were typically seen as variably sized aggregates towards the periphery of the tumour mass, at the edge of necrosis area, or as single cells scattered in the tumour parenchymal area. The percentage of PD-L1 ${ }^{+} \mathrm{M} \varphi s$ was estimated as the percentage of total $\mathrm{CD} 68^{+} M \varphi$, and was scored as negative or positive expression if the percentage was $<5 \%$ or $\geq 5 \%$, respectively. PD-L1-expressing TCs with prominent nucleoli and CD68 negative expression typically showed membranous staining and a variable component of cytoplasmic staining. The proportion of PD-L1-positive TCs was evaluated as the percentage of total TCs, and the sample was scored as negative or positive expression if the percentage was $<5 \%$ or $\geq 5 \%$, respectively. 
Total RNA isolation and quantitative real-time PCR (qPCR)

Total RNA was extracted from archived frozen tissues collected by Bank of Tumor Resource of Sun Yat-sen University Cancer Center using the TRI Reagent solution (Thermo Scientific) according to the manufacturer's protocol. The RNA concentration was determined using a nanodrop ND-1000 spectrophotometer (NanoDrop Technologies, Wilmington, DE, USA). Aliquots $(2 \mu \mathrm{g})$ of total RNA were reverse-transcribed into CDNA using the $5 \times$ All-In-One RT MasterMix (Applied Biological Materials Inc, Richmond, BC, Canada). The specific primers used to amplify the genes are listed in Supplementary Table S2. The PCR was performed in triplicate using SYBR Green real-time PCR Master Mix (TOYOBO, Osaka, Japan) in a Roche LightCycler 480 System (Roche Diagnostics, Pleasanton, CA, USA). The cycle threshold (Ct) values did not differ by more than 0.5 among triplicate samples. $\Delta \mathrm{Ct}$ between target genes and the reference gene $(G A P D H)$ were calculated to perform the data analysis.

\section{Microarray assay and data analysis}

RNA samples from HCC tumours from 24 cases with different PDL1 expression patterns defined by multiplex staining were subjected to a microarray assay. Each PD-L1 expression pattern contained six biological repetitions. Sample amplification, labelling, hybridisation onto Agilent whole human genome oligonucleotide microarrays containing 58341 different oligonucleotide probes (SurePrint G3 Human Gene Expression $8 \times 60 \mathrm{~K}$ Microarray Kit, Agilent), data extraction, and raw data normalisation were performed by Shanghai Biotechnology Corporation (Shanghai, China).

A gene was defined as differentially expressed between different patterns when its mean expression differed by at least twofold between the patterns, with a Student's $t$ tests $p$ value $<$ 0.05 . The differentially expressed genes (DEGs) were separately analysed for GO term enrichment using the online functional annotation tools DAVID 6.7. Representative enriched GO terms with $p$ values $<0.05$ were selected from the $\mathrm{GO}$ biological process terms in DAVID's fat database.

To calculate single-phenotype gene set enrichment, Gene Set Enrichment Analysis (GSEA) software was applied to derive the normalised enrichment score (NES) of the gene signatures using the C5 BP (GO biological process) subset collected by the Molecular Signature Database (MSigDB) version 6.1. Differentially enriched core gene sets between different phenotypes were defined by a nominal $p$ value $<0.05$.

\section{Statistical analysis}

Differences in means for continuous variables were compared using Student's $t$ test or analysis of variance, and differences in proportions were tested by $X^{2}$ test. Kaplan-Meier estimates were calculated and compared using the log-rank test. The Cox proportional hazard regression models were applied to evaluate the prognostic variables for RFS and OS. IBM SPSS (version 21.0; SPSS Inc., Chicago, IL, USA) statistics software was used for all statistical analyses. All data were analysed using two-tailed tests unless otherwise specified, and $p<0.05$ was considered statistically significant.

\section{RESULTS}

HCC tissue samples and PD-L1 expression

Our previous studies showed that PD-L1-expressing monocytes/ macrophages were highly enriched in the peritumoural stroma of a

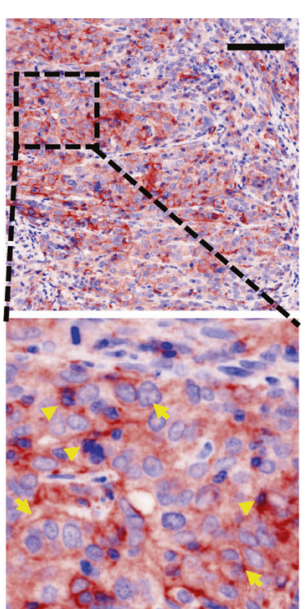

b
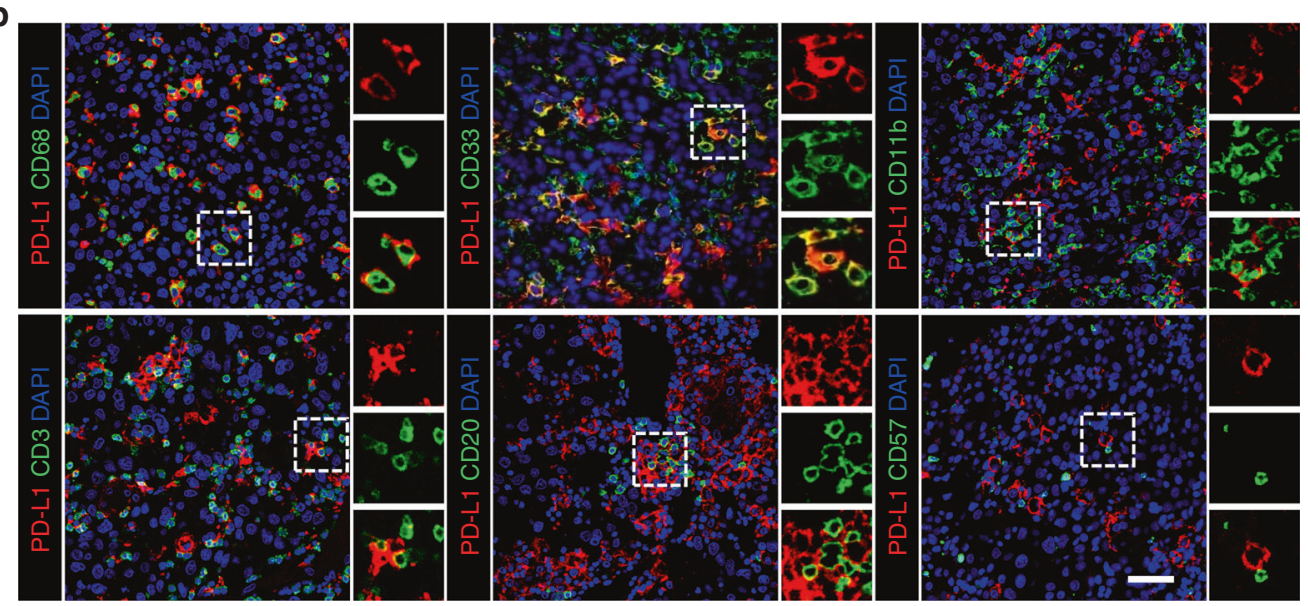

C
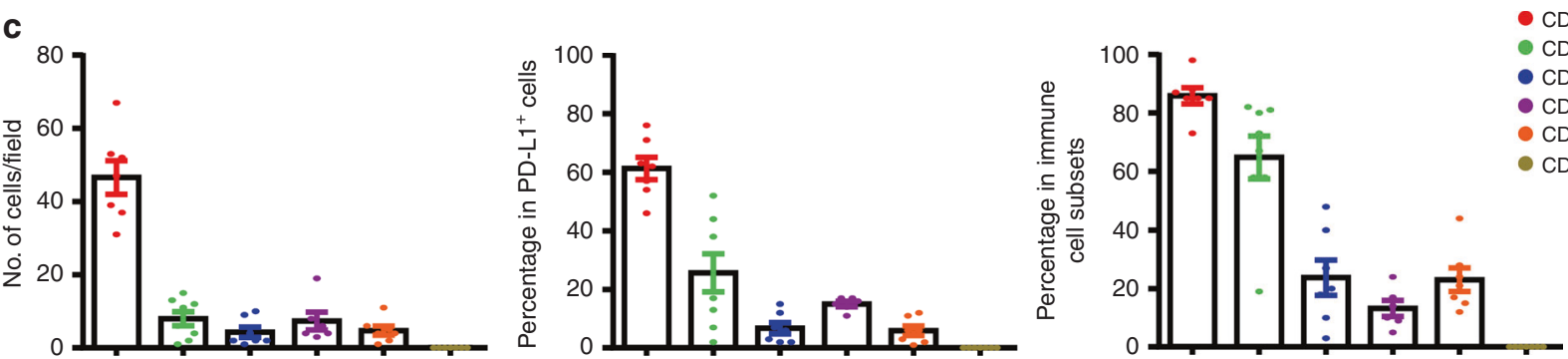

Fig. 1 PD-L1 expression in HCC tumour tissues. a Representative image of PD-L1 (brown) by IHC-TSA staining in HCC tumour tissue. Arrows indicate representative PD-L1 ${ }^{+}$tumour cells, triangles indicate representative PD-L1 ${ }^{+}$immune-cell-like cells. Scale bar, $100 \mu \mathrm{m}$. b Multiplex immunofluorescence staining with the indicated immune cell markers in HCC tumour tissue. PD-L1 staining is shown in red; markers of immune cell markers in green; and DAPI staining in blue. Scale bar, $50 \mu \mathrm{m}$. c Analysis of the percentages of double-positive immune cells in total PD-L1 ${ }^{+}$cells (middle), counts of double-positive cells per field (left), and the percentage of double-positive immune cells within different immune cell subsets (right). Data are expressed as the mean \pm SEM 
a
Pattern 1
$\mathrm{M}^{-} \mathrm{T}^{-}$

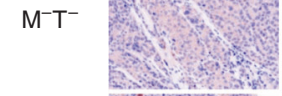

Pattern 2 $\mathrm{M}^{+} \mathrm{T}^{-}$

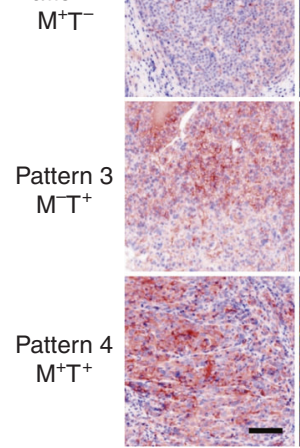

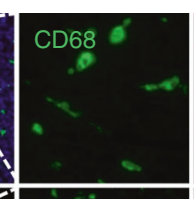
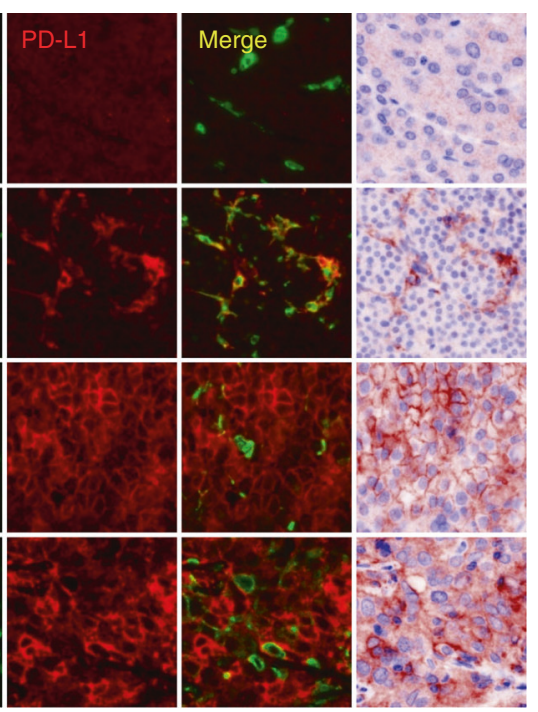

b

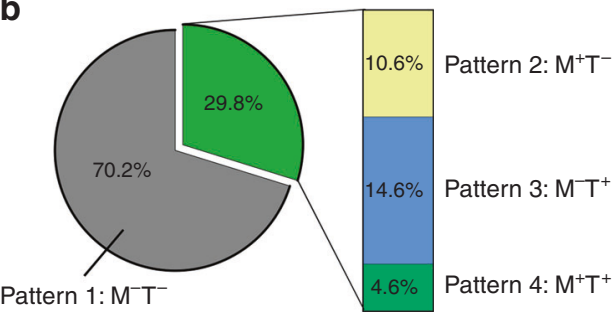

\begin{tabular}{lcc}
\hline Patterns & No. of patients & Percentage $(\%)$ \\
\hline Pattern 1 $\left(\mathrm{M}^{-} \mathrm{T}^{-}\right)$ & 318 & 70.2 \\
Pattern 2 $\left(\mathrm{M}^{+} \mathrm{T}^{-}\right)$ & 48 & 10.6 \\
Pattern 3 $\left(\mathrm{M}^{-} \mathrm{T}^{+}\right)$ & 66 & 14.6 \\
Pattern 4 $\left(\mathrm{M}^{+} \mathrm{T}^{+}\right)$ & 21 & 4.6 \\
\hline
\end{tabular}

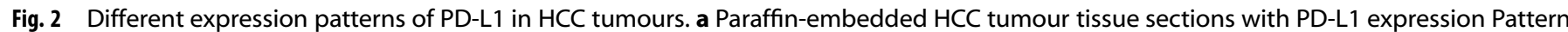
1 ( $\left.\mathrm{M}^{-} \mathrm{T}^{-}\right)$, Pattern $2\left(\mathrm{M}^{+} \mathrm{T}^{-}\right)$, Pattern $3\left(\mathrm{M}^{-} \mathrm{T}^{+}\right)$, and Pattern $4\left(\mathrm{M}^{+} \mathrm{T}^{+}\right)$were subjected to TSA-IHC using anti-human CD68 and anti-human PD-L1

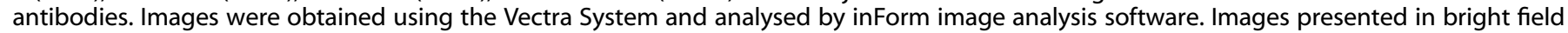

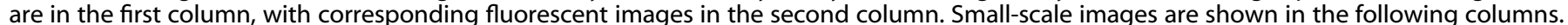
Scale bar, $100 \mu \mathrm{m}$. b Graphs showing the number of patients and the percentage of each pattern

HCC tissues, and could promote tumour progression by impairing T-cell immunity and fostering angiogenesis. ${ }^{29,30}$ Recent studies in other tumour types have revealed that patients with PD-L1 expression on TCs or immune cells in the intra-tumour region showed a better clinical response to anti-PD-1/PD-L1 immunotherapies. ${ }^{16,21-23}$ To fully characterise PD-L1 expression patterns, the clinical significance, and immune status in HCC tumour tissues, 453 intra-tumoural samples from patients with HCC who received curative resection were examined. Most of these patients were male $(400 / 453,88.3 \%)$, and their median age at surgery was 50 years. Among the patients, $91.2 \%$ (413/453) were positive for HBV infection; 96.9\% (439/453) had Child-Pugh score A; $55.2 \%$ (250/453) had a large tumour ( $>5 \mathrm{~cm}$ in diameter), and most patients $(335 / 453,74.0 \%)$ had single tumour foci. Vascular invasion was observed in $11.9 \%(54 / 453)$ of cases. There were 68.7\% (311/453) and 31.3\% (142/453) patients at stage I and II, and stage III and IV, respectively, according to the TNM staging system (7th Edition). In addition, the histological grade of tumour differentiation was defined as I and II (59.8\%, 271/453), and III and IV $(40.2 \%, 182 / 453)$ according to the Edmondson grading system. The detailed clinical and pathological features of the series are summarised in Supplementary Table S1.

Staining of the PD-L1 protein in HCC tissues was performed using TSA-IHC and analysed using the inForm system. Membranous PD-L1 expression could be found on both TCs and immune cells in the intra-tumoural region of HCC tissues (Fig. 1a). Of the 453 samples examined, most samples were weak or negative for PD-L1 expression, only $29.8 \%(135 / 453)$ of the samples were positively stained for PD-L1 expression on TCs or non-TCs. To identify the cell types of the PD-L1 $1^{+}$immune cells, we performed colocalisation studies and calculated the proportion of PD-L1 ${ }^{+}$ cells within each cellular subset in samples of PD-L1 ${ }^{+}$immune cells (Fig. 1b). The majority of PD-L1-expressing cells in these samples $(n=11)$ were $\mathrm{CD}^{+} 8^{+}$myeloid cells, representing $61.29 \pm$ $3.84 \%$ of total PD-L1 ${ }^{+}$cells. T cells and B cells represented only a minority of all PD-L1 ${ }^{+}$cells in HCC tumour tissues $(15.0 \pm 0.93 \%$ and $5.86 \pm 1.63 \%$, respectively; Fig. $1 \mathrm{c})$; PD-L1 ${ }^{+}$NK cells were barely detected in these samples. In addition, we noted that PD$\mathrm{L}^{+}$cells could also express CD33 $(25.63 \pm 6.50 \%)$, but rarely expressed CD11b $(6.71 \pm 1.92 \%)$, which indicated that PD-L1 could be expressed on different subpopulations of myeloid cells in HCC. Collectively, these data indicated that TCs and $\mathrm{CD}^{+} 8^{+}$macrophages were the primary PD-L1-expressing cell types in the intratumour area of HCC tissues.

Different expression patterns of PD-L1 in HCC tumours Recent studies have proposed that different expression patterns of PD-L1 might lead to different biological significance and clinical benefits of anti-PD-1/PD-L1 immune therapies. ${ }^{16,17,24,31,32}$ Therefore, we performed double-colour immunofluorescent staining of PD-L1 with CD68, which could mark most PD-L1 ${ }^{+}$ immune cells in HCC, and divided samples into four groups according to their PD-L1 expression patterns in the intratumoural region (Fig. 2a): (1) Absence of PD-L1 expression on both $M \varphi s(M)$ and TCs (T; Pattern 1: $\left.\mathrm{M}^{-} \mathrm{T}^{-}\right)$; (2) only M $\varphi$ s express PD-L1 (Pattern 2: $\mathrm{M}^{+} \mathrm{T}^{-}$); (3) only TCs express PD-L1 (Pattern 3: $\mathrm{M}^{-} \mathrm{T}^{+}$); and (4) PD-L1 expression on both M $\varphi s$ and TCs (Pattern 4: $\mathrm{M}^{+} \mathrm{T}^{+}$). We then analysed the proportions of the different expression patterns of PD-L1 in the 453 patients with HCC treated by resection (Supplementary Table S1). The incidence rates of PD-L1 expression on M $\varphi$ or TC were $15.2 \%$ (Pattern 2 and 4: $\mathrm{M}^{+} \mathrm{T}^{-}$and $\mathrm{M}^{+} \mathrm{T}^{+}$) and $19.2 \%$ (Pattern 3 and $4: \mathrm{M}^{-} \mathrm{T}^{+}$and $\mathrm{M}^{+} \mathrm{T}^{+}$), 

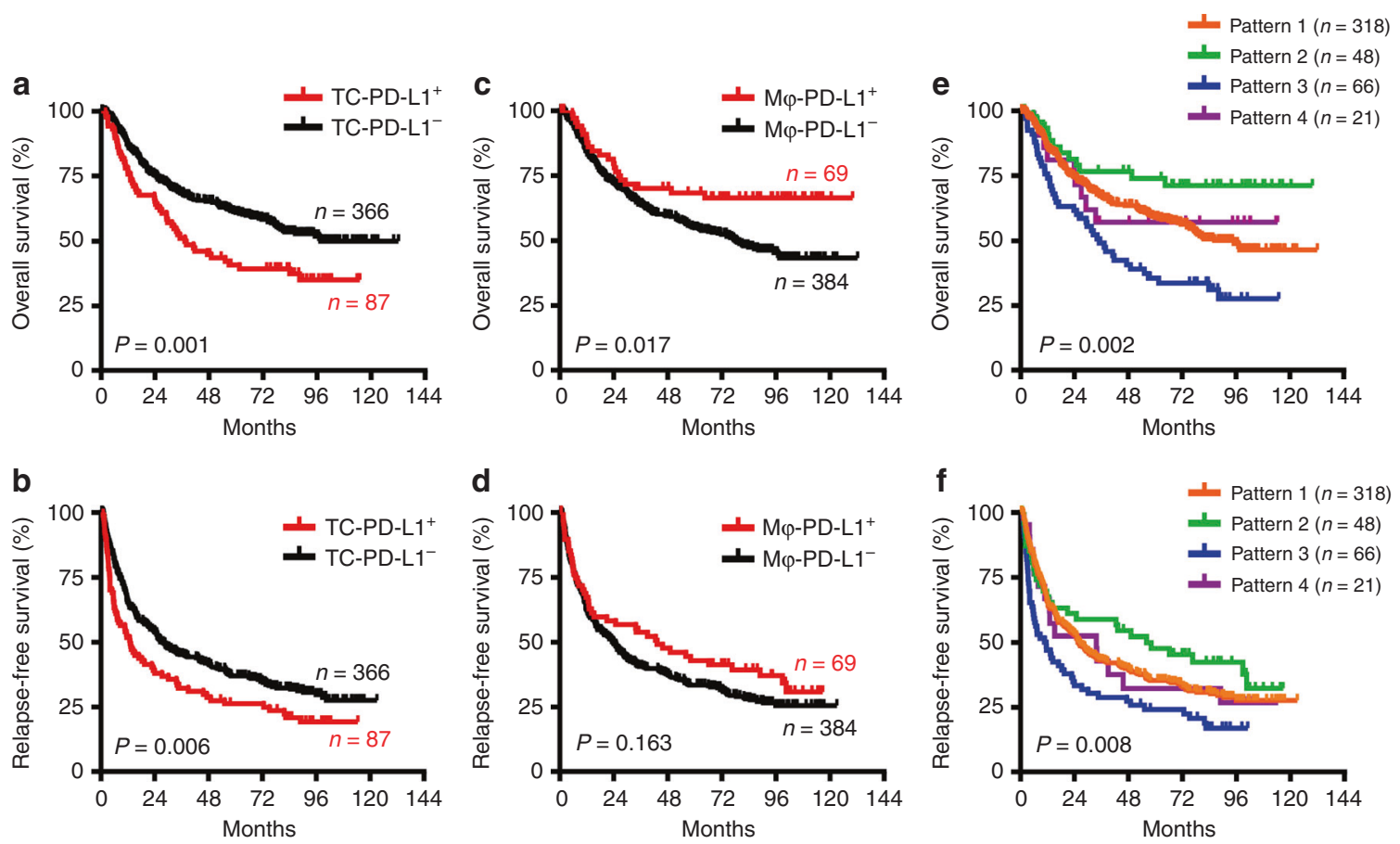

Fig. 3 Differential impact of PD-L1 expression patterns on disease progression in patients with HCC. Cumulative OS (a, c, e) and RFS (b, d, $\mathbf{f})$ of TC-PD-L1 expression (a, b), M $\varphi$-PD-L1 expression (c, $\mathbf{d})$, and combined expression patterns (e, $\mathbf{f})$, were calculated by the Kaplan-Meier method and analysed using the log-rank test

respectively. Only $4.6 \%(21 / 453)$ of the patients had tumours with PD-L1 expression on both M $\varphi$ s and TCs (Pattern 4: $\mathrm{M}^{+} \mathrm{T}^{+}$), while 10.6\% (48/453; Pattern 2: $\left.\mathrm{M}^{+} \mathrm{T}^{-}\right)$and 14.6\% (66/453; Pattern 3: $\mathrm{M}^{-} \mathrm{T}^{+}$) of patients had PD-L1 expression only on M $\varphi s$ or TCs, respectively (Fig. 2b).

Differential impact of PD-L1 expression patterns on disease progression in HCC patients

To evaluate the differential impact of the PD-L1 expression patterns on disease progression in patients with HCC, the correlations between the PD-L1 expression patterns and patients' survival were investigated. Kaplan-Meier survival analysis revealed a striking negative association between the presence of PD-L1expressing TCs and patients' OS and RFS, respectively $(p=0.001$ and 0.006 for OS and RFS, respectively; Fig. 3a, b). Interestingly, the density of PD-L1-expressing M $\varphi s$ was positively correlated with patients' OS ( $p=0.017$; Fig. $3 c)$. Survival analysis showed that patients with the $\mathrm{M} \varphi-\mathrm{PD}-\mathrm{L} 1^{+}$but TC-PD-L1 ${ }^{-}$expression pattern (Pattern 2) had the longest OS (median not reached; 5-year OS, 74.0\%; Fig. 3e) and RFS (median, 56.0 months; 5-year RFS, 48.0\%; Fig. 3f); whereas patients with the M $\varphi-P D-L 1^{-}$but TC-PD-L1 ${ }^{+}$ expression pattern (Pattern 3) had the shortest OS (median, 35.1 months; 5-year OS, 35.0\%; Fig. 3e) and RFS (median, 10.7 months; 5-year RFS, 24.0\%; Fig. 3f).

The presence of PD-L1-expressing M $\varphi s$ was significantly associated with tumour differentiation ( $p=0.003$; Supplementary Table S3). Multivariate analysis revealed that PD-L1 expression on TCs or M $\varphi s$ were both independent prognostic factors for OS (HR $=1.168, p=0.004$ for TC-PD-L1; HR $=0.708, p=0.003$ for M $\varphi$-PD$\mathrm{L} 1$; Table 1). Taken together, these results suggested that the presence of PD-L1 on TCs correlates with tumour progression, whereas the expression of PD-L1 on M $\varphi$ s has a protective role for the prognosis of patients with HCC.

Correlation of PD-L1 expression patterns and immune status of the TME

The expression of PD-L1 (B7-H1) is often induced or maintained by inflammatory cytokines. ${ }^{29,33,34}$ Thus, the upregulation of PD-L1 expression in tumour tissues could be viewed as a reflection of endogenous inflammatory immune responses, but not simply as the dominance of immune suppression. It has been proposed that, based on having either relatively high or low immune cell infiltration, tumours may be immunologically classified into 'hot' (inflamed) or 'cold' (non-inflamed) phenotypes, respectively. ${ }^{35-37}$ Accordingly, clinical evidences showed that patients with an immunogenic TME had a better clinical response to anti-PD-1/PDL1 immunotherapies. ${ }^{16}$ Therefore, we first examined $\mathrm{CD}^{+}{ }^{+}$-cell infiltration in HCC tumours with different PD-L1 expression patterns. As shown in Fig. 4a, b, the density of $\mathrm{CD}^{+}{ }^{+} \mathrm{T}$ cells was significantly higher in tumours with $\mathrm{M} \varphi-\mathrm{PD}-\mathrm{L} 1^{+}$compared with that in $\mathrm{M} \varphi-\mathrm{PD}-\mathrm{L} 1^{-}$tumours $(p<0.0001)$; whereas there was no correlation between TC-PD-L1 expression and $\mathrm{CD}^{+}$T-cell infiltration. We further examined the expression of immune response-related genes using quantitative real-time polymerase reaction (qPCR). As expected, $\mathrm{M} \varphi-\mathrm{PD}-\mathrm{L} 1^{+}$tumours displayed a high level of active immune response-related gene expression (Fig. 4C), including IFNG, GZMB, and PRF1.

To further characterise the immune status of the TME in the HCC tissues with differential PD-L1 expression patterns, we performed gene expression microarray analysis for 24 tumour samples with different PD-L1 expression patterns. Globally, there were 1140 DEGs in the comparison between Pattern 2 and Pattern 1 , and 2031 DEGs when comparing Pattern 4 with Pattern 3 (Supplementary Table S4). Venn diagram analysis revealed that 564 genes were commonly up- or downregulated across these groups, which represented M $\varphi$-PD-L1-related genes (Fig. 5a, top and Supplementary Table S5). These genes were further corroborated by Gene Ontology (GO) analysis, which showed that GO terms for antigen processing and presentation, leucocyte activation, and immune effector process were enriched (Fig. 5b and Supplementary Table S6). Whereas in the analysis of the DEGs between Pattern 3 and Pattern 1, and in Pattern 4 compared with Pattern 2, only 20 common genes were identified, which represented TC-PD-L1-related genes (Fig. 5a, bottom and Supplementary Table S5). Among these 20 common genes, the expression levels of CDK20, AKAP7, AZIN2, LIX1L, OSCP1, and 


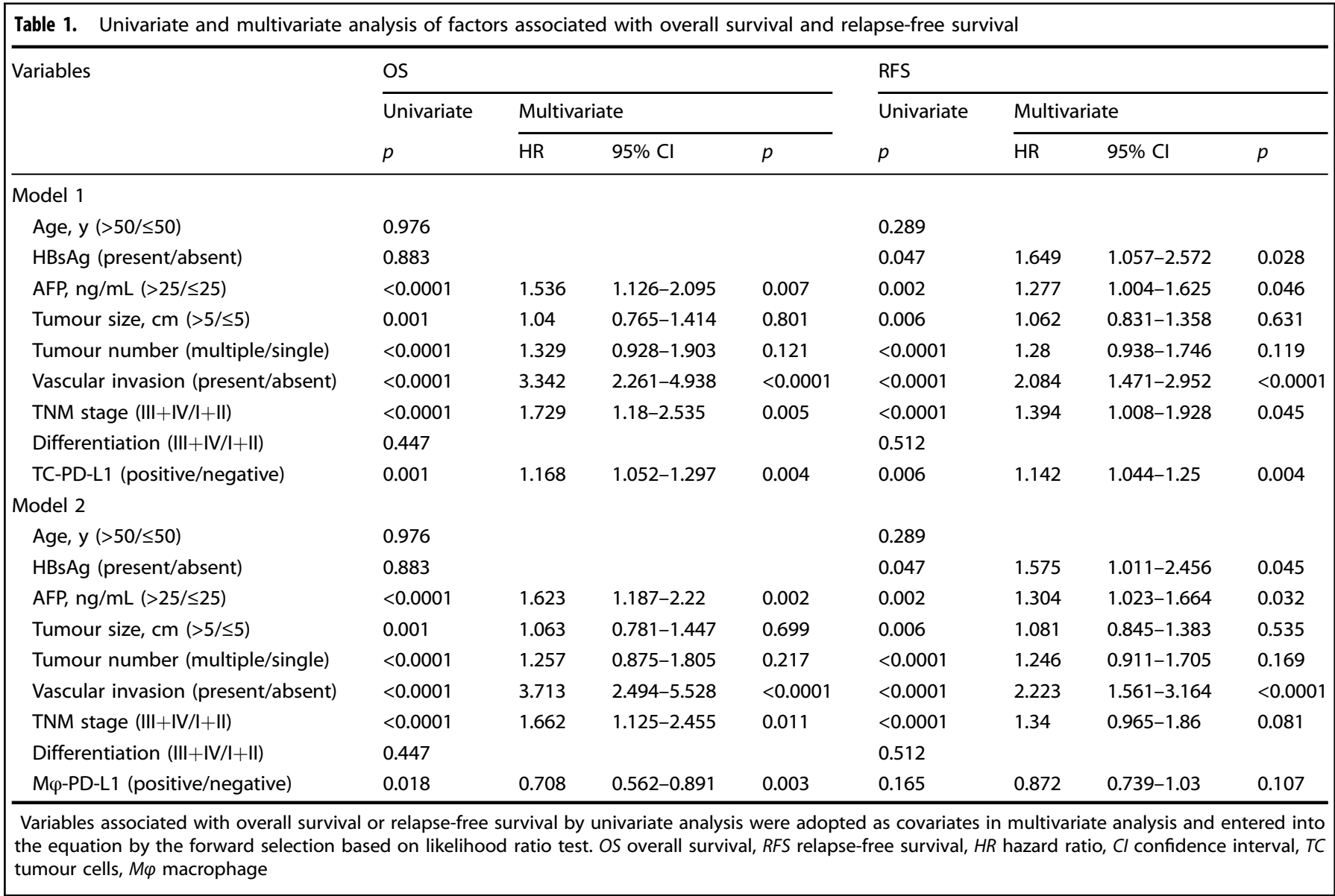

SLFN12 were upregulated, while HLF was downregulated in TC-PD$\mathrm{L}^{+}$tumours (Pattern 3 and Pattern 4).

To determine the differences in cellular process and functional states in HCC tumours with different PD-L1 expression patterns, we performed GSEA using the C5 GO biological process collections in MSigDB. As shown in Fig. 5c, lipid homeostasis, adaptive immune response, quaternary ammonium group transport, and leucocyte-mediated immunity were the most enriched gene sets in Patterns 1-4, respectively (Fig. 5c and Supplementary Table S7 and S8). These data suggested that the identified PD-L1 expression patterns correlated with diverse tumour functional states, and, correspondingly, different TMEs. Taken together, these data suggested that the HCC tissues with $M \varphi-P D-L 1^{+}$patterns correlated with a 'hot' TME, with higher numbers of infiltrating $\mathrm{CD}^{+} \mathrm{T}$ cells and higher levels of immune-related gene expression. This identified PD-L1 expression pattern might serve as a novel method to depict PD-L1 expression to predict the therapeutic responsiveness of anti-PD-1/PD-L1 immunotherapies.

\section{DISCUSSION}

Immune checkpoint therapies targeting PD-1 and PD-L1 have achieved remarkable clinical responses across different tumour types, including HCC; however, the objective response rates varied. Accumulating data have suggested that intra-tumoural expression of PD-L1 could be a potential biomarker to predict the clinical response to anti-PD-1/PD-L1 therapies in patients with melanoma, non-small-cell lung carcinoma, and renal cell carcinoma. ${ }^{12,14,21,38,39}$ To characterise and investigate PD-L1 expression in HCC in detail, we performed double staining using TSA-IHC in 453 patients with HCC. Our results showed that TCs and M $\varphi s$ were the primary PD-L1-expressing cell types in the intra-tumour area of HCC tissues. By TSA-IHC staining of PD-L1 and CD68, we divided patients into four expression patterns: (1) $\mathrm{M}^{-} \mathrm{T}$; (2) $\mathrm{M}^{+} \mathrm{T}^{-}$; (3) $\mathrm{M}^{-} \mathrm{T}^{+}$; and (4) $\mathrm{M}^{+} \mathrm{T}^{+}$according to the expression of PD-L1 on TCs and $\mathrm{M} \varphi$ s. Survival analysis showed the presence of PD-L1 on TCs correlated with tumour progression, whereas the expression of PD-L1 on M $\varphi s$ had a protective role for the prognosis of patients with HCC. Moreover, qPCR and microarray data revealed that $M \varphi$ PD-L1 ${ }^{+}$tumours exhibited an activated immune microenvironment, with high numbers of infiltrating $\mathrm{CD}^{+} \mathrm{T}$ cells and high levels of immune-related gene expression.

Tumour responses to anti-PD-1/PD-L1 immunotherapy are mediated by $T$ cells that had been previously blocked by the PD-1-PD-L1 interaction. ${ }^{40-42}$ Thus, it is reasonable to assume that pre-existing tumour-infiltrating lymphocytes and PD-L1 expression might correlate with clinical response to anti-PD-1/PD-L1 immunotherapy. Clinical trials across different tumour types have confirmed this hypothesis, and the US Food and Drug Administration has approved several IHC test assays to detect PD-L1, which may support clinical decisions for anti-PD-1/PD-L1 therapies. PD-L1 can be expressed on both TCs and immune cells, which have different biological and clinical significance; however, the currently approved assays require a well-trained pathologist to determine the positive cell type and expression level. A recent review of analytical assays reported that a lack of pre-specified criteria could lead to poor intra- and inter-observer concordance in the assessment of PD-L1 expression on immune cells. ${ }^{43}$ In the present study, we found that CD68 could mark most PD-L1 ${ }^{+}$ immune cells in HCC tumours, and that TSA-IHC for CD68 and PDL1 double staining could clearly distinguish PD-L1 expression on TCs and immune cells. These results provided a novel method to determine PD-L1 expression and have a potential application in patient selection for anti-PD-1/PD-L1 therapies. 


\section{C-Q Liu et al.}

a

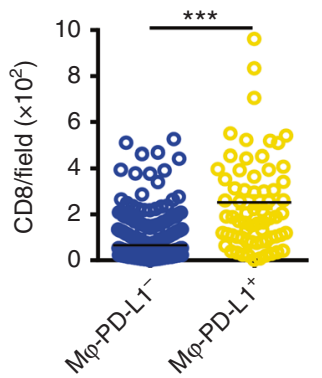

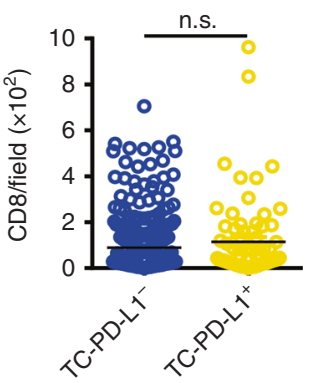

b

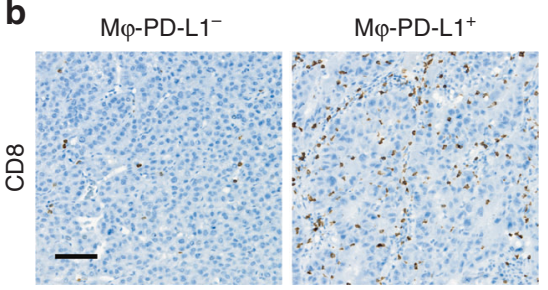

C

Pattern 1

Pattern 2

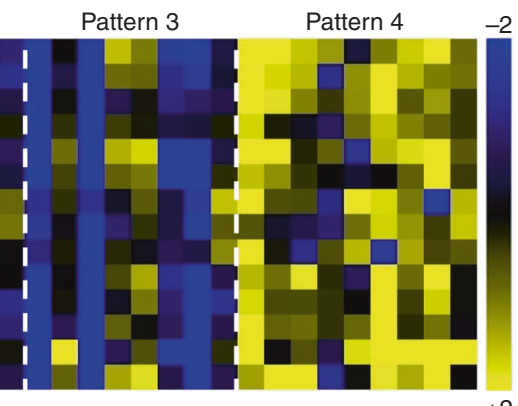

$+2$

Fig. 4 PD-L1 expression on M $\varphi$ s correlated with an active immune microenvironment. a The density of tumour-infiltrating CD8 ${ }^{+} \mathrm{T}$ cells in $\mathrm{M} \varphi$ PD-L1 ${ }^{-}$tumours and M $\varphi-P D-L 1^{+}$tumours (left), and in TC-PD-L1 ${ }^{-}$tumours and TC-PD-L $1^{+}$tumours (right). ${ }^{* * *} p<0.001 ;$ n.s. not significant. $\mathbf{b}$ Representative images of IHC staining of CD8 in sections with M $\varphi-P D-L 1^{-}$and M $\varphi-P D-L 1^{+}$HCC tumour tissue. Images are at $\times 200$ magnification. Scale bar, $100 \mu \mathrm{m}$. c Heatmap showing immune-related gene expression profiles in tumour samples with different PD-L1 expression patterns. The expression levels of different genes were assessed using quantitative real-time PCR. Sets of genes were defined by functional relevance (Th1/Tc1, proinflammation, immune checkpoints, and chemokines)

a

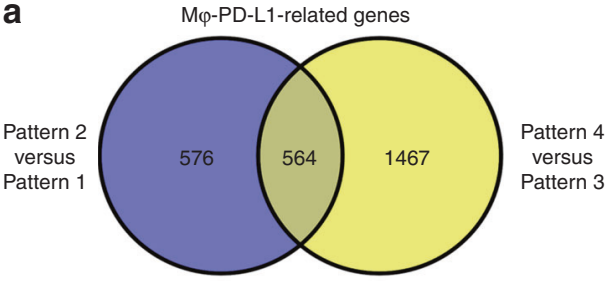

TC-PD-L1-related genes

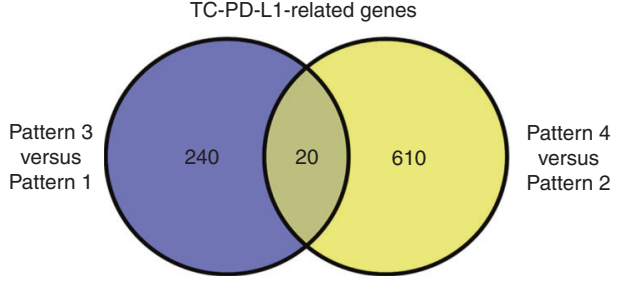

C Most enriched in pattern 1 GO_LIPID_HOMEOSTASIS

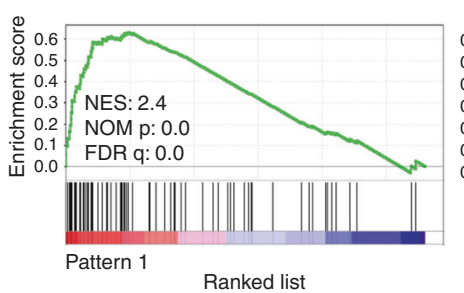

Most enriched in pattern 2 GO_ADAPTIVE_IMMUNE_RESPONSE

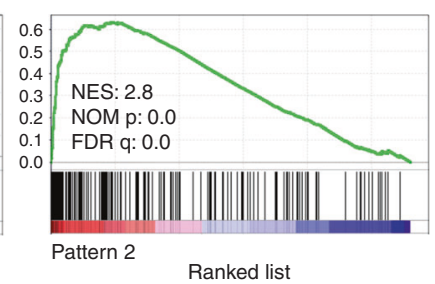

b

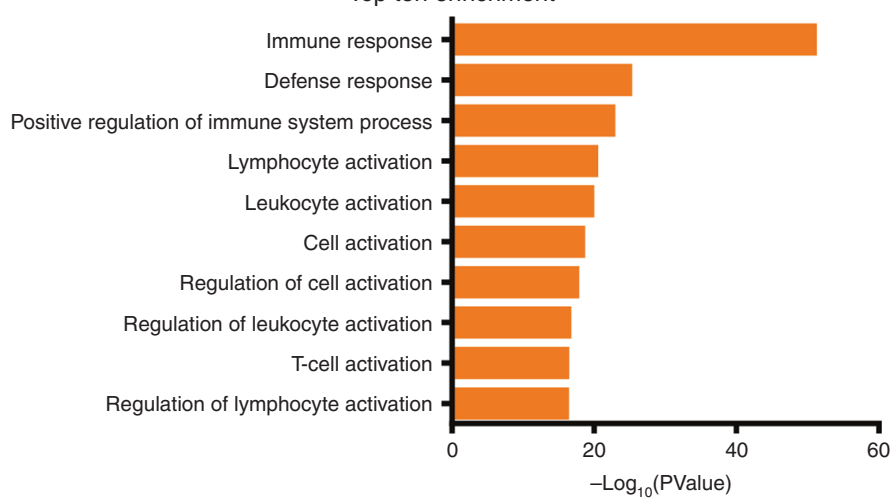

Fig. 5 PD-L1 expression patterns correlated with different TMEs. a Venn diagram analysis of differentially expressed genes between different PD-L1 expression patterns. b Top ten GO terms from the GO enrichment analysis using the common differentially expressed genes between Pattern 2 versus Pattern 1 and Pattern 4 versus Pattern 3. c Gene Set Enrichment Analysis using microarray data of one arbitrary PD-L1 expression pattern versus the other patterns. Selected gene sets are shown. NES normalised enrichment score, NOM $p$ nominal $p$ value, FDR $q$ false discovery rate 
Previous studies have reported the functional roles of PD-L1 expression on $T$ cells and B cells, such as mediating DC maturation by naïve $T$ cells, increasing the survival of effector $T$ cells, and regulating humoral immunity by the interaction between Breg cells with $T_{F R}$ cells. ${ }^{44-46}$ However, the clinical significance of these $\mathrm{PD}-\mathrm{L}^{+}$lymphocytes is currently unknown. Recent studies revealed the essential role of the expression of PD-L1, especially on myeloid cells, in determining the efficacy of PD-L1 blockade. $^{24,47}$ Thus, the evaluation of M $\varphi$-PD-L $1^{+}$tumours should have an important biological implication. In the present study, we showed that $\mathrm{CD} 68^{+}$myeloid cells were the major PD-L1 ${ }^{+}$immune cells in HCC $\left(61.29 \pm 3.84 \%\right.$ of total PD-L1 ${ }^{+}$cells $)$, and were further used to divide the samples into four groups with different PD-L1 expression patterns.

Several groups have attempted to assess the relationship between PD-L1 expression and patients' outcome in HCC; however, these studies mostly examined the overall expression of PD-L1, or simply classified PD-L1 expression on TCs or immune cells by IHC staining. ${ }^{48,49}$ In the present study, CD68 and PD-L1 double staining was applied to determine the positive cell types for PD-L1. Consistent with other reports, TC-PD-L1 expression correlated with poor outcome in patients with $\mathrm{HCC}^{48}$ Interestingly, we found that M $\varphi$-PD-L1 expression was associated with good prognosis in these patients.

Our data further showed that $\mathrm{M} \varphi-\mathrm{PD}-\mathrm{L} 1^{+}$tumours exhibited an activated TME, with high numbers of infiltrating $\mathrm{CD}^{+}{ }^{+}$cells and high levels of immune-related gene expression, including leucocytes chemotaxis, Th1/Tc1 active immune responses, inflammatory cytokines, and immune checkpoints. Microarray analysis confirmed that $\mathrm{M} \varphi$-PD-L1 ${ }^{+}$tumours displayed high expression of genes engaged in the immune response and lymphocyte activation. Thus, the good prognostic value of $M \varphi-P D-L 1$ might be because of the active anti-tumoural TME of the HCC tumours. However, it remains to be determined whether the therapeutic efficacy of PD pathway blockade is similar between patients with $\mathrm{M} \varphi-\mathrm{PD}-\mathrm{L} 1^{+}$tumours and TC-PD-L $1^{+}$tumours in HCC.

Several factors that influence the constitutive PD-L1 expression on TCs have been proposed, such as EGFR activation, PTEN deletions, the MLL1-H3K4me3 axis, and CDK5 disruption. ${ }^{50-53}$ In the present study, we showed that TC-PD-L1 expression did not correlate with T-cell infiltration or the levels of immune response genes. Differential gene expression analysis using a microarray revealed that several genes were commonly upregulated among TC-related genes, such as CDK20. A recent study showed that combined CDK20 inhibition with immune checkpoint blockade might provide more efficient therapeutic effects for patients with HCC. ${ }^{54}$ However, the relationship and underlying mechanism of the association of these genes with PD-L1 expression in HCC is unclear and requires further investigation.

In summary, the expression pattern of PD-L1, as determined by CD68 and PD-L1 double staining, could serve as an important prognostic factor in HCC. This technically simple method could provide a novel approach to detect PD-L1 expression, which might help identify patients who may benefit from anti-PD-1/PDL1 immunotherapies.

\section{AUTHOR CONTRIBUTIONS}

C.-Q.L. and J.X. were responsible for conducting the study, under the supervision of Y.-J.Z. and L.Z., and contributed to the experimental design; C.-Q.L., L.-L.J., Z.-G.Z., X.-J. Y. and J.L. did the experiments and acquired the data; C.-Q.L., J.X. and G.X. performed the data analysis and interpretation; C.-Q.L., J.X., S.-M.Z., and L.Z. wrote and/or revised the manuscript; all authors read and approved the final manuscript.

\section{ADDITIONAL INFORMATION}

Supplementary information is available for this paper at https://doi.org/10.1038/ s41416-018-0144-4.
Competing interests: The authors declare no competing interests.

Funding: This work was supported by the National Key R\&D Program of China under Grant 2017YFA0505803 and Grant 2018ZX10302205; the National Natural Science Foundation of China under Grant 81772536, Grant 91442205, Grant 81730044, and Grant 81572387; and the Fundamental Research Funds for the Central Universities under Grant 171gjc32.

Ethics approval and consent to participate: : This study conformed strictly to the ethical guidelines of the Declaration of Helsinki and was approved by the Research Ethics Committee of Sun Yat-sen University Cancer Center.

Availability of data and material: The authenticity of this article has been validated by uploading the key raw data onto the Research Data Deposit public platform (www.researchdata.org.cn), with the pproval RDD number as RDDB2018000319.

Note: This work is published under the standard license to publish agreement. After 12 months the work will become freely available and the license terms will switch to a Creative Commons Attribution 4.0 International (CC BY 4.0).

\section{REFERENCES}

1. Torre, L. A. et al. Global cancer statistics, 2012. CA Cancer J. Clin. 65, 87-108 (2015).

2. Liver EAFTSOT, Research EOF, Cancer aTO. EASL-EORTC clinical practice guidelines: management of hepatocellular carcinoma. J. Hepatol. 56, 908-943 (2012).

3. Gao, Q. et al. Translational medicine in hepatocellular carcinoma. Front Med. 6, 122-133 (2012).

4. Villanueva, A. et al. New strategies in hepatocellular carcinoma: genomic prognostic markers. Clin. Cancer Res. 16, 4688-4694 (2010).

5. Yang, J. D. \& Roberts, L. R. Hepatocellular carcinoma: a global view. Nat. Rev. Gastroenterol. Hepatol. 7, 448-458 (2010).

6. Llovet, J. M. \& Bruix, J. Systematic review of randomized trials for unresectable hepatocellular carcinoma: chemoembolization improves survival. Hepatology 37, 429-442 (2003).

7. Lee, J. H. et al. Adjuvant immunotherapy with autologous cytokine-induced killer cells for hepatocellular carcinoma. Gastroenterology 148, 1383-1391 e1386 (2015).

8. Topalian, S. L., Drake, C. G. \& Pardoll, D. M. Immune checkpoint blockade: a common denominator approach to cancer therapy. Cancer Cell 27, 450-461 (2015).

9. Chen, L. \& Han, X. Anti-PD-1/PD-L1 therapy of human cancer: past, present, and future. J. Clin. Invest. 125, 3384-3391 (2015).

10. Hodi, F. S. et al. Improved survival with ipilimumab in patients with metastatic melanoma. N. Engl. J. Med. 363, 711-723 (2010).

11. Ansell, S. M. et al. PD-1 blockade with nivolumab in relapsed or refractory Hodgkin's lymphoma. N. Engl. J. Med. 372, 311-319 (2015).

12. Brahmer, J. R. et al. Safety and activity of anti-PD-L1 antibody in patients with advanced cancer. N. Engl. J. Med. 366, 2455-2465 (2012).

13. Powles, T. et al. MPDL3280A (anti-PD-L1) treatment leads to clinical activity in metastatic bladder cancer. Nature 515, 558-562 (2014).

14. McDermott, D. F. et al. Survival, durable response, and long-term safety in patients with previously treated advanced renal cell carcinoma receiving nivolumab. J. Clin. Oncol. 33, 2013-2020 (2015).

15. El-Khoueiry, A. B. et al. Nivolumab in patients with advanced hepatocellular carcinoma (CheckMate 040): an open-label, non-comparative, phase 1/2 dose escalation and expansion trial. Lancet 389, 2492-2502 (2017).

16. Herbst, R. S. et al. Predictive correlates of response to the anti-PD-L1 antibody MPDL3280A in cancer patients. Nature 515, 563-567 (2014).

17. Herbst, R. S. et al. Pembrolizumab versus docetaxel for previously treated, PD-L1positive, advanced non-small-cell lung cancer (KEYNOTE-010): a randomised controlled trial. Lancet 387, 1540-1550 (2016).

18. Budczies, J., Mechtersheimer, G., Denkert, C., Klauschen, F. \& Mughal, S. S. PD-L1 (CD274) copy number gain, expression, and immune cell infiltration as candidate predictors for response to immune checkpoint inhibitors in soft-tissue sarcoma. Oncoimmunology 6, e1279777 (2017).

19. Pardoll, D. M. The blockade of immune checkpoints in cancer immunotherapy Nat. Rev. Cancer 12, 252-264 (2012).

20. $\mathrm{Gu}, \mathrm{T}$. et al. Fine-tuned expression of programmed death 1 ligands in mature dendritic cells stimulated by CD40 ligand is critical for the induction of an efficient tumor specific immune response. Cell. Mol. Immunol. 5, 33-39 (2008).

21. Garon, E. B. et al. Pembrolizumab for the treatment of non-small-cell lung cancer. N. Engl. J. Med. 372, 2018-2028 (2015). 
22. Reck, M. et al. Pembrolizumab versus chemotherapy for PD-L1-positive nonsmall-cell lung cancer. N. Engl. J. Med. 375, 1823-1833 (2016).

23. Rosenberg, J. E. et al. Atezolizumab in patients with locally advanced and metastatic urothelial carcinoma who have progressed following treatment with platinum-based chemotherapy: a single-arm, multicentre, phase 2 trial. Lancet 387, 1909-1920 (2016).

24. Lin, $H$. et al. Host expression of PD-L1 determines efficacy of PD-L1 pathway blockade-mediated tumor regression. J. Clin. Invest. 128, 805-815 (2018).

25. Rimm, D. L. et al. A prospective, multi-institutional, pathologist-based assessment of 4 immunohistochemistry assays for pd-11 expression in non-small cell lung cancer. JAMA Oncol. 3, 1051-1058 (2017).

26. $\mathrm{Xu}$, J. et al. An in situ molecular signature to predict early recurrence in hepatitis $B$ virus-related hepatocellular carcinoma. J. Hepatol. 57, 313-321 (2012).

27. Kuang, D. M. et al. Peritumoral neutrophils link inflammatory response to disease progression by fostering angiogenesis in hepatocellular carcinoma. J. Hepatol. 54, 948-955 (2011).

28. Ding, T. et al. Endothelium-coated tumor clusters are associated with poor prognosis and micrometastasis of hepatocellular carcinoma after resection. Cancer 117, 4878-4889 (2011).

29. Kuang, D. M. et al. Activated monocytes in peritumoral stroma of hepatocellular carcinoma foster immune privilege and disease progression through PD-L1. J. Exp. Med. 206, 1327-1337 (2009).

30. Kuang, D. M. et al. B7-H1-expressing antigen-presenting cells mediate polarization of protumorigenic Th22 subsets. J. Clin. Invest. 124, 4657-4667 (2014).

31. Ribas, A. \& Hu-Lieskovan, S. What does PD-L1 positive or negative mean? J. Exp. Med. 213, 2835-2840 (2016).

32. Lin, H.., ., \& Wei, S.., \& Vatan, L.., \& Kryczek, I.., \& Zou, W.. Relevance of host and tumor P D-L1 expression in PD-L1 and PD-1 blockade. J. Immunol. 198(1 Supplement), 56.55 (2017).

33. Dong, $\mathrm{H}$. et al. Tumor-associated B7-H1 promotes T-cell apoptosis: a potential mechanism of immune evasion. Nat. Med. 8, 793-800 (2002).

34. Lim, S. O. et al. Deubiquitination and Stabilization of PD-L1 by CSN5. Cancer Cell. 30, 925-939 (2016).

35. Sharma, P. \& Allison, J. P. The future of immune checkpoint therapy. Science $\mathbf{3 4 8}$, 56-61 (2015).

36. Zou, W., Wolchok, J. D. \& Chen, L. PD-L1 (B7-H1) and PD-1 pathway blockade for cancer therapy: Mechanisms, response biomarkers, and combinations. Sci. Transl. Med. 8, 328rv324 (2016).

37. Nagarsheth, N., Wicha, M. S. \& Zou, W. Chemokines in the cancer microenvironment and their relevance in cancer immunotherapy. Nat. Rev. Immunol. 17, 559-572 (2017).
38. Topalian, S. L. et al. Safety, activity, and immune correlates of anti-PD-1 antibody in cancer. N. Engl. J. Med. 366, 2443-2454 (2012)

39. Taube, J. M. et al. Association of PD-1, PD-1 ligands, and other features of the tumor immune microenvironment with response to anti-PD-1 therapy. Clin. Cancer Res. 20, 5064-5074 (2014).

40. Tumeh, P. C. et al. PD-1 blockade induces responses by inhibiting adaptive immune resistance. Nature 515, 568-571 (2014).

41. Kleinovink, J. W. et al. PD-L1 expression on malignant cells is no prerequisite for checkpoint therapy. Oncoimmunology 6, e1294299 (2017).

42. Taube, J. M. Unleashing the immune system: PD-1 and PD-Ls in the pre-treatment tumor microenvironment and correlation with response to PD-1/PD-L1 blockade. Oncoimmunology 3, e963413 (2014).

43. Buttner, R. et al. Programmed death-ligand 1 immunohistochemistry testing: a review of analytical assays and clinical implementation in non-small-cell lung cancer. J. Clin. Oncol. 35, 3867-3876 (2017).

44. Talay, O., Shen, C. H., Chen, L. \& Chen, J. B7-H1 (PD-L1) on T cells is required for Tcell-mediated conditioning of dendritic cell maturation. Proc. Natl. Acad. Sci. USA 106, 2741-2746 (2009).

45. Pulko, V. et al. B7-h1 expressed by activated CD8 T cells is essential for their survival. J. Immunol. 187, 5606-5614 (2011).

46. Khan, A. R. et al. PD-L1hi B cells are critical regulators of humoral immunity. Nat. Commun. 6, 5997 (2015).

47. Tang, H. et al. PD-L1 on host cells is essential for PD-L1 blockade-mediated tumor regression. J. Clin. Invest. 128, 580-588 (2018).

48. Calderaro, J. et al. Programmed death ligand 1 expression in hepatocellular carcinoma: relationship With clinical and pathological features. Hepatology 64, 2038-2046 (2016).

49. Gao, Q. et al. Overexpression of PD-L1 significantly associates with tumor aggressiveness and postoperative recurrence in human hepatocellular carcinoma. Clin. Cancer Res. 15, 971-979 (2009).

50. Lu C., et al. The MLL1-H3K4me3 axis-mediated PD-L1 expression and pancreatic cancer immune evasion. J. Natl. Cancer Inst. 109, djw283 (2017).

51. Dorand, R. D. et al. Cdk5 disruption attenuates tumor PD-L1 expression and promotes antitumor immunity. Science 353, 399-403 (2016).

52. Parsa, A. T. et al. Loss of tumor suppressor PTEN function increases B7-H1 expression and immunoresistance in glioma. Nat. Med. 13, 84-88 (2007).

53. Chen, $\mathrm{N}$. et al. Upregulation of PD-L1 by EGFR activation mediates the immune escape in EGFR-driven NSCLC: implication for optional immune targeted therapy for NSCLC patients with EGFR mutation. J. Thorac. Oncol. 10, 910-923 (2015).

54. Zhou, J. et al. Hepatoma-intrinsic CCRK inhibition diminishes myeloid-derived suppressor cell immunosuppression and enhances immune-checkpoint blockade efficacy. Gut 67, 931-944 (2017). 